\title{
UNIFORM BOUNDEDNESS THEOREM FOR A NONLINEAR MATHIEU EQUATION*
}

\author{
BY R. WILHELMSEN (University of Colorado, Boulder)
}

Bolotin [1, Ch. 3-Ch. 6] has derived the equation

$$
f^{\prime \prime}(t)+2 \epsilon f^{\prime}(t)+\Omega^{2}(1-2 \mu \cos \theta t) f(t)+\psi\left(f(t), f^{\prime}(t), f^{\prime \prime}(t)\right)=0
$$

to describe the motion of a parametrically excited pin-ended elastic column. The function $f(t)$ is related to the displacement at time $t$ of the column from its undisturbed position as follows. Let $l$ be the length of the column and $x$ the displacement at height $h$. Then $x=f(t) \sin (\pi h / l)$. In a recent paper Genin and Maybee [2], using an energy function technique, have shown that all solutions are bounded when (1) is of the form

$$
f^{\prime \prime}=-\left(2 \epsilon f^{\prime}+\Omega^{2}(1-2 \mu \cos \theta t) f+\gamma f^{3}+2 \epsilon_{l} f^{\prime} f^{2}+2 \kappa f^{2} f\right) /\left(1+2 \kappa f^{2}\right)
$$

and certain restrictions are placed on the parameters $\epsilon, \Omega, \mu, \theta, \gamma, \epsilon_{l}$, and $\kappa$. In this note a theorem is presented which shows that every solution of (2) has a bound which is independent of the solution's initial values. This result is obtained under less restrictive conditions than those used in [2].

THEOREM. There is a bounded region $A$ in the $x y$-plane such that if $f(t)$ satisfies (2), $f\left(t_{0}\right)=f_{0}$, and $f^{\prime}\left(t_{0}\right)=f_{0}^{\prime}$, then $\left(f(t), f^{\prime}(t)\right) \in A$ for all $t \geq T$ where $T$ is a finite value which depends on $t_{0}, f_{0}$, and $f_{0}^{\prime}$. The conditions placed on the parameters of (2) are:

$$
\epsilon, \gamma>0 ; \epsilon_{l}, \kappa \geq 0 ; \text { and } \epsilon_{l}>0 \text { if } \kappa>0 \text {. }
$$

Proof. Let

$$
\Phi(x, y)=\left(x^{2}+b x y+c y^{2}\right)\left(1+2 \kappa x^{2}\right)+\left(b \epsilon+c \Omega^{2}-1\right) x^{2}+\frac{1}{2}\left(b \epsilon_{l}+c \gamma-4 \kappa\right) x^{4}
$$

where $b$ and $c$ are chosen so that $b>1 / \epsilon$ and $c>\max \left(b^{2} / 4, b /(4 \epsilon),\left(4 \kappa-b \epsilon_{l}\right) / \gamma, \delta\right)$ where

$$
\begin{aligned}
& \delta=0 \quad \text { if } \quad \kappa=0 \text {, } \\
& =b \kappa / \epsilon_{l} \text { if } \kappa>0 \text {. }
\end{aligned}
$$

Since $4 c>b^{2}, x^{2}+b x y+c y^{2}$ is a positive definite form. Also $b \epsilon+c \Omega^{2}-1>0$ and $b \epsilon_{l}+c \gamma-4 \kappa>0$. Hence $\Phi$ is a positive definite function of $x$ and $y$. It is easily seen that, for any constant $C>0$, the contour line $\Phi(x, y)=C$ is a single simple closed curve about the origin.

Let $f(t)$ satisfy (2). Then

$$
\begin{aligned}
\frac{d}{d t}\left[\Phi\left(f(t), f^{\prime}(t)\right)\right]=\frac{\partial \Phi}{\partial f} f^{\prime}+\frac{\partial \Phi}{\partial f^{\prime}} f^{\prime \prime}=-(4 c \epsilon & -b) f^{\prime 2}-4\left(c \epsilon_{l}-b \kappa\right) f^{\prime 2} f^{2} \\
& -b \Omega^{2} f^{2}-b \gamma f^{4}+2 \Omega^{2} \mu \cos \theta t\left(b f^{2}+2 c f^{\prime} f\right) .
\end{aligned}
$$

\footnotetext{
* Received October 15, 1970.
} 
Using $4 c \epsilon-b>0$ we have

$$
\begin{aligned}
\frac{d \Phi}{d t}= & -\left\{(4 c \epsilon-b) f^{\prime 2}+4 \Omega^{2} \mu c \cos (\theta t) f^{\prime} f+\left(4 \Omega^{4} \mu^{2} c^{2} /(4 c \epsilon-b)\right) f^{2}\right\} \\
& -\left\{4\left(c \epsilon_{l}-b \kappa\right) f^{\prime 2}+b \gamma f^{2}-\left[b \Phi^{2}(2 \mu \cos \theta t-1)+4 \Omega^{4} \mu^{2} c^{2} /(4 c \epsilon-b)\right]\right\} f^{2} .
\end{aligned}
$$

Since $|\cos \theta t| \leq 1$

$$
\begin{aligned}
\frac{d \Phi}{d t} \leq & -\left\{(4 c \epsilon-b)^{1 / 2}\left|f^{\prime}\right|-\left(2 \Omega^{2} \mu c /(4 c \epsilon-b)^{1 / 2}\right)|f|\right\}^{2} \\
& -\left\{4\left(c \epsilon_{l}-b \kappa\right) f^{\prime 2}+b \gamma f^{2}-\left[b \Omega^{2}(2 \mu-1)+4 \Omega^{4} \mu^{2} c^{2} /(4 c \epsilon-b)\right]\right\} f^{2} .
\end{aligned}
$$

By examining the possibilities $c \epsilon_{l}-b_{\kappa}=0$ or $c \epsilon_{l}-b \kappa>0$ and $\Omega^{2} \mu=0$ or $\Omega^{2} \mu \neq 0$, it follows from (3) that $d \Phi / d t$ may be nonnegative only in a bounded region of the $x y$ plane. Let $A$ be the region bounded by a contour line of $\Phi$ which encloses in its interior all points at which $d \Phi / d t \geq 0$. Since $d \Phi / d t$ is negative and bounded away from 0 for points not in $A$, it follows that after some finite time, depending on $t_{0}, f_{0}$, and $f_{0}^{\prime}$, the point $\left(f(t), f^{\prime}(t)\right)$ will enter and remain in $A$.

It may also be seen from (3) that the trivial solution has global asymptotic stability when $b \Omega^{2}(2 \mu-1)+4 \Omega^{4} \mu^{2} c^{2} /(4 c \epsilon-b) \leq 0$. This inequality is achieved when $|\mu|$ is sufficiently small.

The author would like to thank Professor Genin for his helpful comments during the preparation of this note.

\section{REFERENCES}

[1] V. V. Bolotin, The dynamic stability of elastic systems, Holden-Day, San Francisco, Calif., 1964

[2] J. Genin and J. S. Maybee, Boundedness theorem for a nonlinear Mathieu equation, Quart. Appl. Math.

$28,450-453(1970)$ 